\title{
Corela
}

Cognition, représentation, langage

HS-1 1 | 2012

RJC Cotexte, contexte, situation

\section{Le contexte revisité}

\section{Catherine Kerbrat-Orecchioni}

\section{OpenEdition}

\section{Journals}

Édition électronique

URL : http://journals.openedition.org/corela/2627

DOI : 10.4000/corela.2627

ISSN : 1638-573X

\section{Éditeur}

Cercle linguistique du Centre et de l'Ouest - CerLICO

\section{Référence électronique}

Catherine Kerbrat-Orecchioni, « Le contexte revisité », Corela [En ligne], HS-11 | 2012, mis en ligne le 02 avril 2012, consulté le 19 avril 2019. URL : http://journals.openedition.org/corela/2627 ; DOI : $10.4000 /$ corela.2627

Ce document a été généré automatiquement le 19 avril 2019

\section{(c) (i) (2)(2)}

Corela - cognition, représentation, langage est mis à disposition selon les termes de la licence Creative Commons Attribution - Pas d'Utilisation Commerciale - Partage dans les Mêmes Conditions 4.0 International. 


\title{
Le contexte revisité
}

\author{
Catherine Kerbrat-Orecchioni
}

1 Dans la présentation du numéro de Pratiques intitulé « Textes. Contextes » et paru en juin 2006, Guy Achard-Bayle déplore que tout en restant « condamné à jouer le rôle ou tenir la place que lui assigne sa définition même: l"arrière plan'", la notion de contexte "a massivement investi les recherches linguistiques ». Les colloques, articles et même numéros de revues consacrés à cette notion ont en effet proliféré ces derniers temps, d'où le titre de cette contribution: «Le contexte revisité" - une fois encore! Titre à connotation légèrement ironique et même auto-ironique car j'ai moi-même eu récemment l'occasion de parcourir de nouveau ce vaste continent ${ }^{1}$. Certes, on n'en a jamais fini avec la question du contexte, thème inépuisable pour les linguistes car la façon d'aborder cet objet épouse les évolutions de notre discipline. Mais on ne peut tout de même pas renouveler tous les cinq ans son discours sur un tel objet : le risque de radotage menace donc.

Deuxième source d'embarras: dans le texte qu'il présente dans ce volume Georges Kleiber, revisitant lui aussi une notion qu'il a abondamment contribué à peaufiner, fait véritablement le tour de la question, sous la forme d'un inventaire des différents problèmes que pose la notion de contexte et sur lesquels on peut adopter des attitudes antagonistes, Kleiber défendant de manière générale une position qui (ré)concilie ces différents points de vue, position avec laquelle je suis d'accord sur toute la ligne. Je me contenterai donc de reprendre en écho les différents points qu'il aborde, en les envisageant sous un angle un peu différent étant donné que nous ne travaillons pas exactement sur les mêmes objets: il s'agira ici essentiellement d'échantillons de "discours en interaction ", réalisés oralement, or il a souvent été signalé que la question du contexte se posait en des termes bien différents selon que l'on avait affaire à des discours écrits ou oraux. En outre, la réflexion sera fatalement tributaire de mon propre environnement de recherche (la mouvance interactionniste et conversationaliste), la façon de traiter la question du contexte illustrant elle-même l'influence du contexte sur la façon dont on appréhende une réalité quelle qu'elle soit (chacun, c'est bien connu, voit midi à sa porte). 


\section{Pour analyser une entité linguistique quelconque, est-il ou non indispensable de prendre en compte certains éléments du contexte?}

\subsection{Deux attitudes opposées}

3 Opposant l'attitude A «hors contexte» (on ne doit s'intéresser qu'aux propriétés intrinsèques de l'entité en question) à l'attitude $B$ « en contexte » (« hors contexte point de salut »), Kleiber renvoie dos à dos ces deux positions, montrant en particulier de façon tout à fait convaincante que « le contexte ne peut pas tout régenter ». Je me contenterai d'ajouter quelques remarques à ce sujet.

(1) À tout prendre, si l'on envisage les formes les plus radicales que peuvent prendre ces deux attitudes, B est encore plus indéfendable que A : on peut tout de même dire des choses sensées sur des énoncés envisagés hors contexte (comme «le chat mange la souris ») alors qu'on ne peut pas dire grand chose sur des énoncés actualisés sans recourir à sa connaissance de la langue. Il semble difficile d'affirmer sérieusement que les formes linguistiques n'ont aucune valeur intrinsèque, leur valeur «émergeant " lors du processus contextualisé de co-construction des énoncés (Kleiber parlant à ce sujet de « contexte démiurge »).

(2) Le problème de la prise en compte du contexte peut être envisagé à deux niveaux, linguistique (le fonctionnement de la langue) et métalinguistique (la description de la langue), mais qui sont étroitement liés : la réponse à la question « quelle place l'analyse linguistique doit-elle accorder aux considérations sur le contexte ? » découle directement de la réponse à la question: «quelle place le contexte joue-t-il dans le fonctionnement des unités linguistiques?»

(3) La réponse à ces deux questions est fonction du type d'unités auxquelles on s'intéresse, mais aussi et surtout du fait qu'on les envisage plutôt par rapport au système de la langue ou dans leur actualisation discursive; et dans le deuxième cas, elle est fonction du type de discours auquel on a affaire.

On sait qu'au cours de son évolution la linguistique n'a cessé de s'intéresser à des unités de plus en plus étendues, et corrélativement de les envisager de plus en plus dans leurs réalisations concrètes, et corrélativement encore de tenir de plus en plus compte du contexte. Il n'empêche que quelles que soient les unités sur lesquelles on travaille, l'analyse peut se focaliser plutôt sur le système abstrait des règles qui sous-tendent leur fonctionnement, ou plutôt sur leur fonctionnement "situé ». Il va de soi que dès que l'on travaille sur le discours la prise en compte du contexte est indispensable. Mais nous commencerons par montrer que cette prise en compte s'impose déjà au niveau de la langue: cela vaut par exemple pour les formes « déictiques ", dont la définition implique la prise en compte de certains ingrédients de la situation de communication ; mais on peut le voir avec d'autres types d'unités, comme les actes de langage.

\subsection{L'exemple des actes de langage}

5 Telle qu'elle a été formulée au départ, la théorie « austino-searlienne » des speech acts a pour objectif de décrire le système des différents actes de langage et familles d'actes existant dans une langue donnée, avec leurs différents types de marqueurs et de valeurs 
illocutoires. Comme il n'y a de correspondance biunivoque entre signifiants et signifiés (la synonymie et la polysémie sont généralisées), on peut s'attendre à ce que le contexte joue un rôle important dans le fonctionnement de ce système : on le verra avec le problème des actes de langage indirects; mais le contexte intervient aussi à d'autres niveaux, en particulier celui des « conditions de réussite » de l'acte de langage.

\subsubsection{Les conditions de réussite}

Elles sont toutes de nature contextuelle, pouvant porter sur le locuteur A, le destinataire B, ou l'« état de choses » (dans le cas par exemple d'un ordre tel que « Ferme la porte », il faut que l'état de chose ne soit pas déjà réalisé au moment de l'énonciation de l'acte, c'està-dire que la porte soit ouverte). Mais ces conditions externes laissent aussi, pour le grand bénéfice de l'analyste qui peut ainsi les débusquer, des traces dans les énoncés sous la forme de "répliques» de la part du destinataire («Mais elle est fermée!») ou de précautions de la part de locuteur («Si la porte est ouverte, ferme-la »). C'est ce que nous allons voir à partir du cas du vœeu, acte dont la réussite est soumise à une série de conditions bien précises et passablement tyranniques concernant l'« état de choses souhaité »; par exemple en ce qui concerne un énoncé votif tel que «Bon voyage ! »:

(1) Que l'événement sur lequel porte le vœu doive effectivement se produire, c'est-

à-dire en l'occurrence qu'il y ait un voyage en vue.

Réplique de B :

«Finalement je reste à Lyon tout l'été. »

Précaution de A (afin de devancer et neutraliser une réplique potentielle) :

"Si tu décides de partir, bon voyage!»

Autres exemples de précautions similaires s'appliquant à d'autres formules votives :

«Bonnes vacances, si vous en prenez »

[Dans l'avion à l'atterrissage] :

«Bonne journée, et pour les passagers en transit, bonne continuation de votre voyage! » (le segment " pour les passagers en transit » servant à circonscrire le sous-ensemble des destinataires pour lesquels le vœu est pertinent).

(2) Que ce puisse être une bonne chose.

7 Réplique correspondante : «Je déteste voyager. »

8 Précaution: «Je sais que tu n'aimes pas beaucoup voyager mais je te souhaite quand même un bon voyage. »

Autres exemples de réplique ou de précaution similaire :

«Bonne balade! - Par ce temps?»

(À quelqu'un qui vient de subir un deuil familial) : «Bon week end, bien qu'il ne doive pas être pour vous bien joyeux... ».

Difficile en effet de formuler des vœux à propos d'événements intrinsèquement «mauvais» (comme un enterrement). Dans certains cas, la transgression de la condition de réussite peut susciter une interprétation ironique :

« Aujourd'hui j'ai huit heures de cours, la galère ! - Bon ben bonne galère! »

(3) Que le vœeu ne soit pas "superflu ", c'est-à-dire que l'état de choses favorable ne doive pas se réaliser à coup sûr (on peut voir dans cette condition une sorte de résidu de la valeur primitive du vœu, reposant sur l'idée que l'on peut vraiment modifier un état de choses, donc agir sur le monde, par des moyens purement langagiers).

Réplique : « Mais c'est toujours bon les voyages!»

Précaution : «Inutile de te souhaiter bon voyage!»

9 Autres exemples : 
"Je suis très heureuse que tu aies réussi ton concours. Inutile donc de te souhaiter une bonne année 2009 : elle ne pourra être pour toi qu'heureuse et fructueuse. » "Je te souhaite bon courage mais je sais que tu n'en manques pas. " «Ce petit message en début d'année pour te souhaiter plein de bonnes choses comme bonheur, sérénité, joie, succès (mais est-ce bien nécessaire ?) » Les vœux de bonne année se prêtent particulièrement bien à toutes sortes de jeux sur ces conditions de réussite, par exemple :

«Je vous souhaite jeunesse, beauté... - Mais j’ai déjà tout ça ! "

"Alors qu'est-ce qu'on vous souhaite? - Tout! - Mais vous avez tout! -

Presque!»

11 Notons que ces vœux de nouvel an sont soumis à une condition temporelle qui peut elle aussi donner lieu à un commentaire plus ou moins ludique, par exemple dans un message posté à l'extrême fin du mois de janvier : "Vite vite avant l'échéance je te souhaite une très bonne année 2010 ». Mais tous les vœux sont soumis à des conditions concernant le moment de formulation du vœu (T1) par rapport au moment de réalisation prévue de l'événement souhaité (T2).

(4) Conditions temporelles

- Que T1 soit antérieur à T2.

Réplique :

«Mais je suis rentrée hier!»

Précaution:

«Si tu n'es pas encore partie, bon voyage!»

Autres exemples :

«Bon appétit, à moins que vous ayez déjà mangé !»

14 Dans le cas des échanges par courrier électronique, le fait que la temporalité ne soit pas partagée et que l'on ne sache pas à quel moment le destinataire va lire le message peut imposer des précautions particulières :

«Bonne soirée, si vous êtes encore devant votre écran. » / «Bonne nuit, si tu n'es pas encore couchée. »

"Je suis sans mail jusqu'à lundi inclus (je pars en Normandie tout à l'heure). -

Bonne Normandie, si tout à l'heure n'est pas encore passé !»

- Que A et B ne doivent pas se revoir avant T2.

Réplique :

«Mais on se revoit demain!»

Précaution :

«Si on ne se revoit pas d'ici ton départ, bon voyage!»

Autre exemple : «Je te dis bon week-end puisque tu n'as pas cours demain.»

17 Cette condition peut entrer en conflit avec le principe voulant que la formulation du vœu, car il risquerait sinon d'être oublié le moment venu, ne soit pas non plus trop éloignée du moment de réalisation de l'événement souhaité, ce qui peut donner lieu à des échanges tels que :

«Bonnes vacances! - Mais c'est dans un mois! - Je sais mais on ne va sûrement pas se revoir d'ici là...»

\subsubsection{Les actes de langage indirects}

Soit les deux énoncés suivants : 
(1) « Tu peux me passer le pot de confiture?»: selon que le pot en question se trouve à portée de main ou dans un endroit plus difficilement accessible, l'énoncé sera interprété plutôt comme une requête indirecte (demande d'un faire) ou comme une simple question (demande d'un dire, sur laquelle peut éventuellement venir se greffer, mais seulement dans un deuxième temps, une requête explicite ou implicite).

(2) «J'aimerais bien une bière bien fraîche » : selon que l'énoncé est prononcé dans un bistrot à l'intention du serveur ou en plein désert à l'intention d'un compagnon d'infortune tout aussi assoiffé et démuni de la moindre canette, l'énoncé fonctionnera comme une requête indirecte ou comme une simple assertion (il peut aussi dans un autre contexte être interprétable comme une proposition).

Lorsque ces énoncés valent pour une requête il s'agit d'une requête indirecte conventionnelle, dans la mesure où la valeur indirecte découle d'une règle de dérivation illocutoire telle que dans le premier cas : « toute question sur la capacité d'accomplir une certain acte adressée à une personne dont il est évident qu'elle a cette capacité vaut pour une requête d'accomplir ce cet acte »; et dans le deuxième : "Toute assertion d'un désir adressée à quelqu'un qui a la possibilité (et a fortiori l'obligation comme c'est le cas en contexte commercial) de satisfaire le désir en question vaut indirectement pour une requête de satisfaire ce désir ».

20 Entre les actes indirects conventionnels et non conventionnels la distinction est en fait graduelle, comme l'ont montré un certain nombre d'analyses ${ }^{4}$. On ajoutera que cette gradualité repose sur différents facteurs :

- le degré de grammaticalisation de la formulation comme support d'une valeur indirecte, par exemple : pour une demande d'information, « Tu peux me dire si/où $X$ ? » est beaucoup plus systématiquement interprété comme "Dis-moi si/où $\mathrm{X}$ " que "Je ne sais pas si/où $\mathrm{X}$ ", d'après Anquetil (2009) qui envisage en outre trois autres structures intermédiaires permettant de réaliser indirectement cet acte de langage ;

- la présence éventuelle de marqueurs de dérivation illocutoire, qui peuvent confirmer ou même dans certains cas constituer la valeur indirecte; par exemple dans le cas d'une question rhétorique un adverbe comme « vraiment » («Pensez-vous vraiment que X ?»); ou dans le cas d'une requête, le morphème de politesse «s'il te plaît» («Tu peux attraper le pot de confiture s'il te plât ?»): comme la requête est un acte plus fortement impositif que la question, l'adoucisseur s'impose davantage en cas de requête, on peut donc inférer la valeur de requête de sa présence dans l'énoncé ; ou encore un terme d'adresse («Papa j'ai faim » / «La porte est ouverte Martine »), qui accentue la pression sur le destinataire et oriente donc l'énoncé dans la même direction. Ces marqueurs ou indices peuvent aussi être de nature prosodique ou mimo-gestuelle (par exemple, un index pointé transformant en sommation de

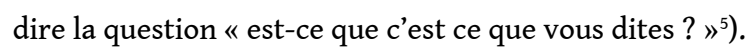

- Quant aux effets du contexte ils sont eux aussi graduels, le pot de confiture ou la bière bien fraîche pouvant être plus ou moins accessibles et disponibles. Contexte qui est susceptible de jouer une rôle tout à fait similaire à celui des facteurs linguistiques précédemment envisagés : on peut parler d'une équivalence fonctionnelle entre les facteurs externes et internes, et par exemple formuler un principe général tel que : «Plus le degré de conventionalisation de la structure responsable de la valeur indirecte est fort, moins le contexte est nécessaire à son actualisation; plus le degré de conventionalisation de la structure est faible, plus le contexte est nécessaire pour faire émerger la valeur dérivée » - les deux cas limites étant représentés d'un côté par celui du véritable «trope illocutoire", pour lequel le contexte peut tout au plus avoir une fonction de blocage du mécanisme dérivationnel (notons 
toutefois que même dans ce cas l'équivalence entre la formulation directe et indirecte est dénotative mais non connotative, puisque «Tu peux fermer la porte» est plus poli que «Ferme la porte ») ; et de l'autre par celui d'une dérivation totalement non conventionnelle dont je fournirai un exemple personnel : si je laisse à mon compagnon un mot sur lequel il est écrit «C'est mercredi!", il comprendra automatiquement qu'il doit penser à acheter Lyon Poche, en vertu d'un raisonnement qui implique un savoir contextuel très local (le fait que c'est le mercredi que parait cet outil indispensable aux Lyonnais pour programmer leurs activités culturelles de la semaine).

On pourrait multiplier les exemples de cette équivalence fonctionnelle. En voici deux encore, rencontrés dans nos corpus :

(1) Dans les commerces la vendeuse accueille généralement la cliente par un «Madame bonjour/ » accompagné d'une mélodie montante : dans ce cas la valeur de question («Que désirez-vous?») qui vient s'ajouter à la salutation est supportée par un marqueur intonatif. Mais le même énoncé peut aussi être prononcé avec une mélodie descendante, sans perdre pour autant cette valeur de question: c'est alors le seul contexte qui se charge de la faire émerger, c'est-à-dire la connaissance que possèdent les participants du "script » caractéristique de ce type d'événement communicatif.

(2) Autre rituel d'ouverture : la question-de-salutation « (comment) ça va? », acte hybride penchant tantôt du côté de la salutation, tantôt du côté de la question ${ }^{6}$. Là encore les facteurs qui interviennent dans cette orientation peuvent être de nature "interne" (emplacement, nature exacte de la formulation...) mais aussi « externe » : nature du site ainsi que des savoirs sur l'état de santé du destinataire, comme l'illustre cet exemple de François Mitterrand interviewé par Bernard Pivot le 15 avril 1995 peu avant la fin de son mandat, précédant de huit mois seulement sa disparition :

BP. - Monsieur le Président, comment allez-vous?

FM. - Jusqu'au mois de mai prochain - ce n'est pas loin -, jusqu'au 7 mai, c'est une question d'État. Mais après ce sera une question privée. [silence] J'essaierai de tenir jusque là.

Tous ces exemples montrent la nécessité d'incorporer certaines données contextuelles à la formulation des règles qui sous-tendent le fonctionnement des actes de langage (on peut par exemple énoncer pour rendre compte du cas précédent un principe tel que : « Si une question de salutation est adressée par $\mathrm{A}$ à $\mathrm{B}$ qui a des problème de santé ou de quelque autre nature, lesquels problèmes sont supposés connus de $\mathrm{A}$, alors la formule n'est pas une simple salutation mais elle conserve une valeur question »). Ces règles font partie intégrante de la "grammaire» des actes de langage et de la compétence pragmatique des locuteurs. Lorsque la description se situe au niveau du système de la langue, les facteurs contextuels pertinents sont formulés en termes de contextes-types; lorsque la description porte sur des énoncés actualisés dans un contexte particulier, elle fera émerger des valeurs et des effets supplémentaires inédits, ainsi qu'on va le voir dans les rubriques suivantes.

\section{Contexte défini comme « ensemble d'éléments qui influencent l'interprétation » vs contexte comme « pur environnement »}

On voit mal comment les entours d'une unité $\mathrm{X}$ pourraient être sans influence sur $\mathrm{X}$, aussi bien du point de vue de la production de X par A (c'est-à-dire de l'ensemble de ses choix 
discursifs) que de l'interprétation de X par B. La définition du Nouveau Petit Robert (2007) le dit fort bien :

CONTEXTE.

1. Ensemble du texte qui entoure un mot, une phrase, un passage et qui sélectionne son sens, sa valeur ${ }^{7}$.

2. Ensemble des circonstances dans lesquelles s'insère un fait.

Cette définition distingue deux types de contextes qui sont en réalité tous deux susceptibles d'avoir de l'influence sur l'unité envisagée, même si le dictionnaire ne le mentionne qu'en 1: on reviendra sur cette distinction entre différentes sortes d'environnements. Mais pour l'heure, intéressons-nous à la diversité des influences qu'ils peuvent exercer sur un segment discursif quelconque, et en particulier sur les effets possibles du contexte sur l'interprétation : comme ils sont fort bien décrits et classés par Kleiber (lever une ambiguité, faire rentrer dans le rang un emploi en principe déviant, compléter l'interprétation en produisant certains effets de sens...), on se contentera de lui faire écho en signalant que ces différents cas de figure peuvent fort bien être illustrés par le fonctionnement des actes de langage, ainsi que l'on vient déjà d'en avoir un aperçu. Nous poursuivrons la réflexion avec un échantillon de discours mentionné dans notre précédent article de 2009, mais que nous allons exploiter ici de façon un peu différente. Il s'agit de l'extrait suivant d'un débat mettant en présence Nicolas Sarkozy et Jean-Marie Le Pen au cours de l'émission télévisuelle 100 minutes pour convaincre (France 2, 20 novembre 2003) :

1- OM monsieur Sarkozy alors euh Jean-Marie Le Pen président du Front National est avec nous euh vous allez débattre ensemble bonsoir monsieur Le Pen

2- LP bonsoir

3- OM voilà (.) prenez place (.) monsieur Le Pen [...] qu'est-ce que vous avez à dire/ à monsieur Sarkozy

4- LP ASP ${ }^{8}$ ben j'voudrais d'abord dire/ que ma tâche n'est pas facile ici puisque je sui : :s (.) le représentant/ d'une catégorie (.) politique rare/ dans notre pays/ c'est celle des parias $\backslash$ (..) [...] ASP monsieur le ministre de l'Intérieur/ vous me donnez l'impression : :/

[ASP]

5- NS [bonsoir/] monsieur Le Pen

6- LP bonsoir/ bonsoir monsieur eh j'ai dit bonsoir en arrivant/ ASP mais euh vous étiez inclus collectiv- dans mon bonsoir collectif [ASP]

7- NS [dans ma] cage d'écureuil

8- LP : tout à fait (.) je oui :// (.) eh bien/ écoutez/justement $\backslash$ je je dois dire/ que :

[je trouve que vous ressemblez à à un écureuil]

10a. NS : [méfiez-vous ça peut être sympathique un écureuil]

11a. LP : ASP c'est ça et l'é- l'écureuil [est sympa-]\&

10b. NS: [méfiez-vous]

11b. LP : \&-thique/ il a même du panache/ n'est-ce pas mais il tourne dans sa cage/

ronde/ (.) en se donnant lui/l'impression qu'il fait/ beaucoup/ ASP mais alors qu'il

n'avance pas du tout

Décrivons sommairement ce qui se passe dans cette séquence : Sarkozy est sur le plateau depuis un bon moment déjà (ayant été confronté à divers interlocuteurs) quand Le Pen fait son entrée. L'animateur Olivier Mazerolles le salue, Le Pen salue à son tour (à la ronde, d'où l'absence de tout terme d'adresse), puis au lieu de s'adresser à Sarkozy conformément à la consigne, il se lance aussitôt dans une diatribe (que nous n'avons pas fait figurer ici) contre le monde politico-médiatique qui le traite comme un "paria ». Sarkozy le laisse faire son petit numéro durant plus d'une minute, et au moment même où Le Pen s'apprête, après ces préliminaires, à passer à l'attaque contre son interlocuteur 
(fin du tour 4), celui-ci l'interrompt avec un «Bonsoir monsieur Le Pen» tout à fait imprévu. Stoppé dans son élan, Le Pen se voit administrer par Sarkozy un coup de semonce et une petite leçon de savoir-vivre. Contraint de retourner la salutation, il se sent aussi tenu de justifier son comportement. Manifestement déstabilisé, il produit à la fin de 6 un "raté » suivi d'une "réparation» ("vous étiez inclus collectiv- dans mon bonsoir collectif »), ce qui n'est guère son style. À peine remis en selle, il se voit de nouveau interrompu par Sarkozy en 7, avec «dans ma cage d'écureuil» qui vient se greffer de façon d'ailleurs assez artificielle sur le terme « inclus " pris dans un autre sens que celui qu'il possède en 6 , et qui est une allusion au fait que juste avant l'entrée de Le Pen, a été projeté un bref extrait d'un film où l'on voit le président du Front National déclarer lors d'une conférence de presse que «Nicolas Sarkozy fait semblant d'avancer mais il tourne en rond comme un écureuil en cage ».

Essayons de cerner le rôle du contexte dans l'interprétation des deux segments « bonsoir monsieur Le Pen » en 5 et « dans ma cage d'écureuil » en 7.

(1) Alors que le «bonsoir monsieur Le Pen » de OM est une salutation « pure » - c'est-àdire que le segment reçoit en discours la valeur, ni plus ni moins, qui lui est attribuée par la langue -, le même segment exactement va recevoir dans la bouche de Sarkozy la double valeur d'une salutation et d'un reproche, la valeur de reproche découlant d'un raisonnement tel que : en entrant en interaction avec moi vous auriez dû me saluer, or vous ne l'avez pas fait, donc je le fais à votre place pour vous donner l'exemple (la salutation de NS est en réalité polyphonique : c'est à la fois celle de Sarkozy et une anticipation de celle qu'il sollicite de Le Pen). Notons que la deuxième valeur vient se greffer sur la première : il est impossible de comprendre la valeur de reproche sans prendre en compte au préalable la salutation sur laquelle vient se greffer le reproche, cet exemple illustrant autant la robustesse des valeurs conventionnelles qui s'attachent aux formes linguistiques (en l'occurrence le lexème «bonsoir ») que leur malléabilité contextuelle. Bien d'autres valeurs, que l'on peut dire interactionnelles, viennent en effet se surajouter à ces deux valeurs illocutoires : déstabilisation de l'adversaire et dérèglement de l'échange ; invalidation du petit discours qui précède (et qui n'avait effectivement pas lieu d'être : Le Pen était censé s'adresser directement à Sarkozy, dont le reproche porte aussi sur ce point ); double attribution d'éthos (NS affiche un éthos poli tout en construisant de son adversaire l'image d'un grossier personnage); négociation des places (LP se trouve mis par NS mis en position basse, étant contraint de se justifier comme un enfant pris en faute)... La plupart de ces valeurs sont plus ou moins traitées dans l'enchaînement : ainsi en 6 LP réagit-il successivement à la salutation et au reproche qui sont amalgamés dans la première partie de la paire adjacente ; il réagit aussi du même coup à l'accusation de manque de savoirvivre; et il réagit à l'entreprise de déstabilisation par le bredouillement de 6 aussitôt « réparé ».

En ce qui concerne la valeur de reproche, on ne saurait la considérer comme relevant véritablement de l'indirection conventionnelle. Mais on peut remarquer d'une part que les salutations-reproches ne sont pas rares dans notre environnement quotidien', et d'autre part qu'elles peuvent être ramenées à un principe tel que : «Lorsque B produit une salutation à l'intention de A qui aurait dû saluer le premier mais ne l'a pas fait, cette salutation peut fonctionner comme un reproche ». Dans ce cas particulier, l'actualisation 
de cette valeur (ainsi que des valeurs interactionnelles qui en découlent) est favorisée par des facteurs de nature diverse :

- La prosodie est nettement différente de celle qui accompagne en 1 le «bonsoir monsieur Le Pen » de l'animateur (il ne s'agit donc pas en réalité du «même» segment exactement): l'intonation de Sarkozy est fortement montante, ce qui lui confère un caractère emphatique et théâtral. Cet élément relève pour nous de la matérialité discursive, c'est-à-dire du " texte » lui-même ${ }^{10}$. Il en est de même pour la petite mimique de triomphe et de satisfaction par laquelle Sarkozy accueille la salutation réactive de Le Pen, sorte d'indice rétroactif de la valeur de reproche ;

- L'emplacement de la salutation joue dans cette affaire un rôle décisif : elle est en effet différée par rapport au tout début de l'échange puisque Le Pen a déjà commencé à parler depuis plus d'une minute lorsqu'elle survient. Mais la situation est ici bien spéciale: le « dilogue » NS-LP est en effet enchâssé dans un polylogue qui a débuté en amont, or LP a effectivement (ainsi qu'il le rappelle en guise de justification de son comportement) salué à la ronde en pénétrant sur le plateau. On peut donc se demander si le renouvellement de la salutation, adressée cette fois au seul Sarkozy, s'impose en la circonstance. C'est pour le moins douteux : notre système rituel est à cet égard flottant, mais il est permis de penser que cette salutation relève de l'« hyperpolitesse » plus que de la politesse. Elle est pour le moins inattendue, d'où son effet déstabilisant sur Le Pen, qui de toute évidence ne s'y attendait pas. La valeur de politesse de la salutation (qui est en principe un acte «flatteur " pour la face de l'interlocuteur) est en tout cas plus qu'annulée par ces atteintes à la face de LP que constituent le reproche, l'accusation de grossièreté et l'entreprise de déstabilisation : c'est en fait un sale tour que NS joue à LP, mais sous les dehors d'un comportement poli et même hyperpoli - Sarkozy gagne donc sur tous les tableaux : même s'il débusque la ruse, l'auditoire ne peut qu'applaudir.

- L'interprétation de cette séquence implique donc la connaissance de certaines normes de politesse, qui relèvent de la compétence socio-pragmatique des locuteurs. Elle implique aussi et surtout la prise en compte du "genre interactionnel » dans lequel s'inscrit cette séquence, à savoir un débat dans lequel il s'agit avant tout de triompher de l'adversaire, ce qui va favoriser l'interprétation «malveillante» de ce «bonsoir » qui dans un contexte différent (une rencontre amicale par exemple) pourrait être perçu tout autrement.

29 (2) Le rôle du contexte est plus décisif encore dans le cas de T7: «dans ma cage d'écureuil » est tout bonnement incompréhensible (même si l'on comprend le sens des mots qui composent ce syntagme) si l'on ne possède pas certaines informations contextuelles - mais de quel type de contexte s'agit-il exactement?

\section{Les différents types de contextes}

Quels que soient le cadre théorique et la terminologie adoptés, on admet très généralement qu'il convient de distinguer deux grands types de contextes, le contexte interne (contexte " linguistique », « discursif », " endogène » ou " séquentiel », parfois appelé également "cotexte», ce qui rend ambigu le terme de "contexte» qui tantôt s'oppose à «cotexte» et tantôt l'englobe) vs externe («extralinguistique» ou « exogène »). Cette distinction correspond aux deux subdivisions de la définition du Petit Robert mentionnée plus haut : 
CONTEXTE.

1. Ensemble du texte qui entoure un mot, une phrase, un passage [...]

2. Ensemble des circonstances dans lesquelles s'insère un fait.

31 Dans les deux cas, le contexte envisagé peut être plus ou moins étroit (microcontexte) ou large (macrocontexte). On appelle généralement « situation » le contexte externe étroit ( setting, cadre spatio-temporel généralement immédiatement accessible aux participants à l'interaction), par opposition au macrocontexte social ou culturel.

Ces distinctions posent évidemment toutes sortes de problèmes.

(1) Sur quelle base distinguer le «texte » (c'est-à-dire le segment soumis à l'analyse, qui peut être de dimension variable) et son "cotexte», étant donné qu'ils sont intrinsèquement de même nature ? C'est le moment de rappeler que le contexte est une notion relative: un élément n'est pas contexte « en soi » mais « de quelque chose». La frontière entre texte et cotexte est elle-même relative - à une décision de l'analyste, qui va «focaliser » son travail descriptif sur tel ou tel segment, mais au fur et à mesure que l'analyse progresse, ce qui était « cotexte » devient «texte » et inversement ${ }^{11}$. C'est aussi le moment de revenir sur le cas de «la cage d'écureuil », qui n'est compréhensible que par rapport à une déclaration antérieure de Le Pen. En tant qu'extraite d'une interaction antérieure, cette déclaration a plutôt le statut d'un intertexte. Mais à partir du moment où elle est reproduite et enchâssée dans cette nouvelle interaction que constitue l'émission télévisuelle, elle se trouve convertie en cotexte : on voit que la distinction entre cotexte et intertexte repose elle aussi sur une décision de l'analyste concernant la façon dont il découpe les unités soumises à l'analyse (le problème de savoir s'il s'agit de la même unité globale ou d'une unité différente se posant pour les interactions orales au même titre que pour les textes écrits).

(2) Entre le cotexte et le contexte externe, la distinction est en principe moins problématique puisqu'il s'agit d'unités de nature radicalement hétérogène (matériau discursif vs non discursif). Mais le problème se déplace au niveau "métadescriptif ", prenant la forme de la question suivante: dans quelle mesure l'analyse peut-elle ou doitelle recourir à des informations externes? Cette question fait aujourd'hui débat parmi les interactionnistes - un débat qui n'est pas sans rappeler celui qui fit rage il y a quelques décennies en linguistique textuelle entre ceux qui ne considéraient pas comme un crime de convoquer certaines informations externes au texte lui-même, et les partisans d'une description "immanente » (pour qui le texte fournit toutes les informations nécessaires et suffisantes pour son interprétation). De façon similaire, on peut aujourd'hui opposer les ethnographes de la communication (Gumperz en tête) qui considèrent que l'analyste doit commencer par récolter le plus d'informations possible sur le site étudié; et les adeptes de l'analyse conversationnelle (conversation analysis) qui estiment au contraire qu'il faut se contenter des informations "endogènes », au nom des arguments suivants que je résume rapidement :

- Si l'on commence par dire que l'événement communicatif à étudier se déroule, par exemple, dans une classe ou un commerce cela risque de «biaiser » l'analyse, car dans de tels sites il peut se passer tout autre chose qu'un cours ou une transaction commerciale. Cet argument renvoie à une critique de la conception déterministe de la relation texte-contexte (les caractéristiques du premier étant vues comme automatiquement entraînées par la nature du second). Mais personne ne prétend que dans une classe ou un commerce ne peuvent avoir lieu que des échanges didactiques ou commerciaux: le contexte ce n'est pas seulement le cadre matériel (une assemblée de quartier peut fort bien, par exemple, se dérouler dans une 
école ou un bistrot), c'est aussi et surtout le genre dont relève l'interaction avec son format participatif, ses statuts et ses rôles, son «script » et la succession des tâches et des activités qui le composent... Les participants ont bien évidemment toujours la possibilité de sortir, à leurs risques et périls, du cadre assigné ; de subvertir les règles du genre ou d'inverser les rôles, mais les effets de tels comportements ne se peuvent évaluer que par rapport aux normes qu'ils reconfigurent. Il peut se faire qu'un professeur se comporte comme un élève et inversement, qu'un client se comporte comme un commerçant et inversement, mais on peut se demander ce que vaudrait dans un tel cas une description qui se contenterait de décrire ce que fait l'acteur sans se donner la peine de le confronter à ce qu'il est.

- Un deuxième argument consiste à rappeler que le contexte est un ensemble infini d'éléments qui ne sont pas tous interactionnellement pertinents, les éléments pertinents étant toujours « indiqués » par les « membres » eux-mêmes : en un instant $\mathrm{T}$, les participants sélectionnent tel ou tel aspect du contexte qu'ils vont « exposer » et rendre significatif par leur conduite ; c'est-à-dire que les données contextuelles pertinentes sont en quelque sorte internalisées par le travail des membres, si bien que d'après Schegloff (1997) repris par Mondada (2006: 117) :

Le contexte n'est jamais une dimension "extérieure " à l'action qui viendrait l'éclairer, l'expliquer, ou la structurer; le contexte émerge réflexivement de la manière dont les participants organisent leur action,

les guillemets suggérant le fait que dans cette perspective, l'opposition « externe / interne » se dissout complètement, au profit des seules considérations internes.

En d'autres termes le contexte est entièrement construit dans l'interaction, d'après Mondada toujours (1998: 249) ${ }^{12}$ :

Le contexte n'est pas donné mais construit par les participants dans leur effort pour le rendre disponible.

Plusieurs objections pourraient être faites à cet argument, la principale étant que les analyses ne cessent de démentir l'idée que tous les éléments pertinents du contexte sont forcément inscrits dans le texte. On pourrait en donner bien des exemples. Nous nous contenterons ici d'en emprunter un à l'étude de Marion Sandré (2009: 86) sur les interruptions dans les Questions au Gouvernement : à propos de la réaction «Très bien! » d'un député, produite en interruption, elle note que l'on pourrait être tenté d'interpréter comme ironique cette réaction si l'on ignore que l'interrupteur est UMP, c'est-à-dire du même bord que l'interrompu. En l'absence de tout indicateur prosodique clair, il est indispensable pour interpréter correctement ce qui se passe de tenir compte de cette « information préalable », que possèdent d'ailleurs tous les participants «actifs » à l'interaction.

- Ce qui nous suggère une dernière objection à cette attitude descriptive (last but not least) : elle entre en totale contradiction avec le principe selon lequel l'analyse doit être faite « du point de vue des membres ", puisqu'elle met entre parenthèses tous les savoirs préalables dont ils disposent et qu'ils mobilisent lors de leur activité interprétative, savoirs qui sont en grande partie partagés par les participants à l'interaction - on ne voit vraiment pas au nom de quoi ceux-ci feraient l'« effort » de « rendre disponible » aux co-participants les éléments d'un contexte dont ils ont pour la plus grande part connaissance (et encore moins à un analyste dont ils ignorent le plus souvent jusqu'à l'existence). 


\section{Contexte pour l'observateur-analyste vs contexte pour les participants (ou « membres »)}

\subsection{Adopter «le point de vue des membres »}

Si l'on admet le dogme de l'analyse conversationnelle selon lequel l'analyste se doit d'adopter le point de vue des membres (attitude dite "émique», en un sens qui n'a d'ailleurs qu'un rapport lointain avec la façon dont Pike oppose les points de vue « étique » et " émique »), on est aussitôt renvoyé à la question de savoir ce qu'il convient d'entendre exactement par cette formule. Adopter le point de vue des membres, c'est pour nous tenter de reconstituer la façon dont ils interprètent mutuellement les énoncés qu'ils échangent, et sur cette base, projettent leur activité discursive. Mais cette reconstitution ne va pas de soi étant donné que l'analyste a au départ sur l'interaction un «point de vue » radicalement différent de celui des membres - il exerce son activité a posteriori, dans un lieu et une temporalité très différents, avec des objectifs et un outillage également très différents, c'est-à-dire dans un contexte tout différent. Répétons-le une fois encore : le contexte n'est pas le même pour tout le monde, c'est-à-dire pour les différents participants et à plus forte raison pour les participants et pour l'analyste.

(1) En ce qui concerne le contexte linguistique : comme le rappelle Kleiber (ici même, pour les participants «en action ", en un moment $\mathrm{T} 1$ du déroulement du discours il n'y a pas encore d' « environnement linguistique complet », le seul contexte existant étant le contexte antérieur. Le "côté droit», lui, est "à venir", il n'existe qu'à l'état de potentialité même si certaines anticipations sont possibles du fait des règles de « dépendance conditionnelle » et du caractère « routinisé » de certains échanges.

Alors que pour les participants le texte comme le cotexte ont un caractère intrinsèquement dynamique, l'activité de l'analyste s'exerce généralement sur des unités achevées, conçues comme entourées d'un segment à gauche et d'un segment à droite. En ce sens l'analyste est "suréquipé informativement ", ce qui entraîne un certain nombre de conséquences descriptives que Kleiber passe en revue. En analyse du discours, on peut certes s'efforcer de suivre au fil du texte, qu'il soit de nature monologale ou dialogale, la façon dont il se construit pas à pas, c'est-à-dire tour après tour lorsqu'il s'agit d'échantillons de discours en interaction (ce qui n'interdit pas à l'analyste, ni aux participants d'ailleurs, de faire après coup une sorte de bilan de ce qui s'est passé et de proposer une interprétation d'ensemble de la séquence soumise à l'analyse). C'est vers une description de ce type qu'il faut tendre, sans se faire d'illusion toutefois car ce que l'on sait de l'ensemble de l'ensemble influence inévitablement l'analyse séquentielle :

Il ne me semble pas possible de faire comme si je n'avais pas «le tout » [...] sous les yeux. Je ne peux qu'assumer le fait d'avoir écouté maintes fois la cassette, transcrit l'interaction, avec l'écoute si particulière que cette opération suppose, en être imprégnée, décrire ce qui se passe à l'instant T1 en sachant ce qui s'est passé à l'instant T2. (Traverso $2003: 30$ )

Il est bien évidemment impossible de décrire le déroulement d'une interaction « en temps réel », en même temps qu'on la découvre. La description effectuée par l'analyste est fatalement optimisée par rapport à ce que perçoivent les participants de l'événement dans lequel ils se trouvent engagés (de la même manière que s'agissant d'un texte écrit, la lecture d'un « expert » ne se confond pas avec celle d'un lecteur ordinaire). 
41 (2) Le problème se pose en des termes bien différents s'agissant du contexte externe, puisque sur ce plan, «c'est l'observateur qui se trouve désavantagé ». Dans le cas des textes de fiction toutefois, par exemple dans le cas d'une pièce de théâtre, l'analyste est logé à la même enseigne, non certes que les personnages, mais que ces autres destinataires «officiels» que sont les lecteurs/spectateurs; on peut donc penser que généralement, l'auteur s'arrange pour livrer à ces destinataires l'essentiel des informations contextuelles nécessaires à la compréhension du texte ${ }^{13}$. Mais lorsqu'il s'agit d'interactions authentiques, l'analyste est eavesdropper selon la terminologie de Goffman, c'est-à-dire un «intrus » qui n'est pas prévu au programme; il ne dispose donc pas de toutes les informations contextuelles pertinentes (sauf s'il a lui-même participé à l'interaction, ce qui pose d'autres problèmes), ni même de toutes les informations textuelles (problème des «bruits » éventuels, absence des données mimo-gestuelles si l'enregistrement est simplement audio, et s'il s'agit d'un enregistrement vidéo, problème des restrictions dues au cadrage et aux prises de vue).

Certes, l'analyste peut reconstituer un certain nombre de ces informations contextuelles grâce aux "indices de contextualisation ", expression que nous utilisons de façon un peu différente de Gumperz pour renvoyer à tout de qui, dans le texte, fournit des informations sur le contexte, permettant à l'analyste de le reconstituer en partie, mais en partie seulement. Car on l'a dit précédemment, toutes les informations contextuelles pertinentes ne sont pas inscrites dans le texte, et inversement toutes les informations contextuelles inscrites ne sont pas également pertinentes ("pertinent» étant entendu comme qualifiant ce dont on a vraiment besoin pour comprendre «ce qui se passe»). En outre, ces informations n'ont généralement pas le même statut pour les participants et pour l'analyste : pour reprendre l'exemple d'un énoncé tel que «Bonnes fêtes de Noël » analysé dans notre article de 2009, il apparaît que la formule votive n'a pas pour but de « rendre disponible » le fait que Noël approche, mais bien plutôt de manifester de bonnes dispositions envers l'interlocuteur (afin d'achever dans une ambiance détendue une interaction plutôt mal engagée dans l'exemple en question). Si cet énoncé "exhibe » le contexte pertinent, c'est seulement pour l'analyste qui ne disposerait pas au préalable de cette information et qui peut l'extraire "par la bande ", grâce au présupposé lié à la condition de réussite du vœu. Mais les membres, eux, le savent bien que Noël approche. Si l'on veut adopter le point de vue des membres il faut donc traiter cette information contextuelle comme une information préalable simplement rappelée au cours de l'interaction, mais non comme une information émergente, ce qu'elle n'est éventuellement que pour l'analyste.

Le cas des débats et interviews médiatiques est en quelque sorte intermédiaire entre celui des discours fictionnels et celui des conversations naturelles : il s'agit bien d'interactions authentiques (entre des personnes réelles et non des personnages imaginaires), mais où l'analyste peut être considéré comme un membre de l'ensemble des auditeurs même si son objectif est bien particulier ; lesquels auditeurs doivent être considérés comme des membres à part entière de l'événement communicatif : même s'ils sont généralement confinés dans le rôle de récepteurs, ce sont en fait les destinataires principaux des échanges qui se tiennent dans le studio ou sur le plateau. Ce type de situation illustre bien le fait que le contexte n'est pas le même pour tous les participants. Il convient surtout à cet égard d'opposer les participants " actifs » aux auditeurs de la radio et aux spectateurs de la télévision: les premiers n'ont même pas d'accès visuel au studio; quant aux seconds, ils ne voient pas tout ce qui se passe sur le plateau, étant entièrement soumis 
aux décisions des cameramen et aux normes de cadrage - par exemple, pour le débat de l'entre-deux-tours des Présidentielles entre Ségolène Royal et Nicolas Sarkozy (dorénavant « Sarkolène »), la règle était de ne faire apparaitre à l'écran que le candidat ayant la parole, la consigne spécifiant explicitement que «l'autre ne sera pas filmé pour ne pas dénaturer par l'image le propos du candidat» (ce qui sous-entend que le comportement de l'écouteur peut avoir une incidence sur l'interprétation que l'on fait des propos du parleur). Ce comportement constitue donc une donnée contextuelle non accessible aux spectateurs de ce débat mais accessible au locuteur, et qui peut avoir sur celui-ci des effets déstabilisants (ou encourageants, mais la chose est plus rare); par exemple lorsque Sarkozy farfouille dans ses papiers durant la grande tirade de la «saine colère » de Royal, comme on peut le constater car on a droit à ce moment-là à un plan large (si les plans de coupe sont prohibés il arrive en effet, mais c'est assez exceptionnel, que les deux protagonistes apparaissent simultanément à l'écran).

On peut secondairement opposer les débatteurs aux animateurs (sans parler des membres de l'équipe de tournage) : ces différents acteurs n'ont pas tous exactement le même "point de vue» sur la scène interactionnelle; ils n'ont pas non plus les mêmes préoccupations ni les mêmes objectifs : avant tout soucieux de faire respecter l'agenda et l'équilibre $\mathrm{du}$ temps de parole, les animateurs écouteront et interprèteront essentiellement sous cet angle les propos qui s'échangent sous leur houlette - un peu à l'instar des modérateurs de conférences, dont l'attention se porte tout particulièrement sur les marqueurs de structuration du discours du conférencier qu'ils ont pour mission de "modérer ", guettant les indices permettant de savoir où il en est et s'il s'approche ou non de sa conclusion.

\subsection{Une illustration : le débat "Sarkolène ", séquence dite de la " saine colère »}

Du long débat (2h40) qui vit s'affronter le 2 mai 2007 Ségolène Royal et Nicolas Sarkozy, un épisode surtout est resté dans les mémoires, et restera sans doute dans les annales relatives aux débats présidentiels. Il survient aux deux tiers du débat, alors qu'est censée se clore la séquence sur la politique économique et sociale. Sarkozy vient de vanter le dispositif du "droit opposable», et pour contrer les sarcasmes de Royal il prend l'exemple (un « exemple qui va peut-être vous toucher », annonce-t-il - il ne croit pas si bien dire...) des enfants handicapés qui se voient refuser l'accès dans les écoles, dont il conclut (et l'intonation comme la mimique viennent souligner la valeur conclusive de cette sorte de péroraison) que c'est ce genre de mesure «qui fait la différence entre la vieille politique et la politique moderne». Les animateurs (Poivre d'Arvor et Arlette Chabot) félicitent alors les débatteurs d'avoir « réussi la prouesse de... » mais on ne saura jamais exactement de quoi car Royal les interrompt avec un solennel «attendez j'ai quelque chose à dire » qui sonne comme un coup de théâtre. Ce qu'elle a à dire, c'est qu'elle est scandalisée par l'« immoralité politique » dont fait preuve Sarkozy en tenant un discours «larmoyant » sur les handicapés alors que c'est justement lui qui a "cassé » le plan « handiscole » qu'elle avait elle-même mis en place lorsqu'elle était ministre de l'enseignement scolaire. Elle l'accuse donc longuement d'un « écart entre le discours et les actes » et conclut son réquisitoire en se déclarant « très en colère » :

$\mathrm{SR}:[. .$.$] je suis très en colère \backslash($.$) et les parents et les familles qui vous [ent-\&$

NS : [calmez-vous

SR : \&et les parents [non je ne me calmerai pas 


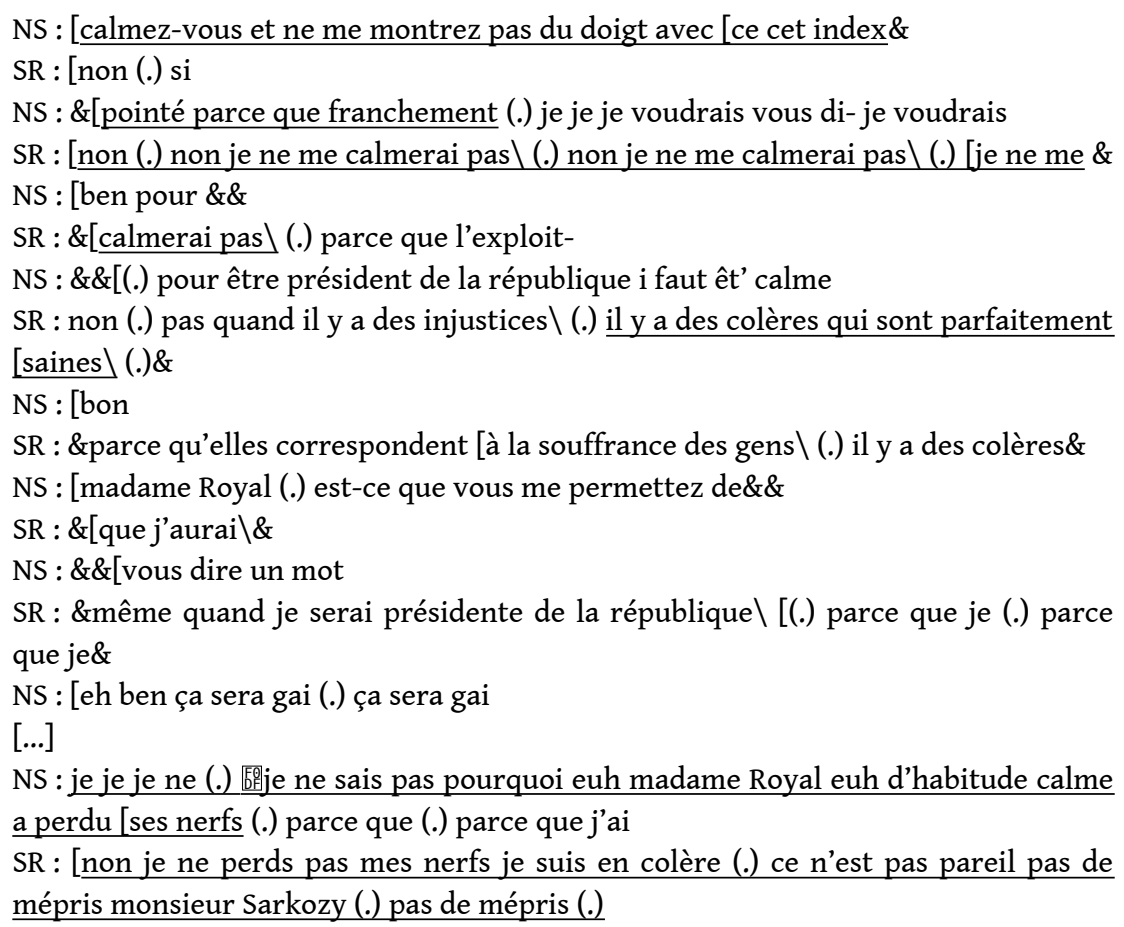

S'ensuit une longue négociation (la séquence dure plus de huit minutes alors que la modératrice a accordé à Royal " un mot puis on enchaîne ») concernant l'état émotionnel de SR, laquelle revendique une « saine colère » quand Sarkozy ne cesse de l'accuser d'être " énervée », de " perdre ses nerfs » et d'être « sortie de ses gonds ». NS s'emploie à établir une équivalence entre la colère et l'énervement, alors que SR s'obstine à répéter que « ce n'est pas pareil », l'assimilation de la colère (qui quand elle est « saine » est une émotion noble) à un vulgaire énervement relevant pour elle du « mépris ». La négociation piétine et s'éternise, offrant diverses variations sur le thème : «Ne vous énervez pas - Je ne suis pas énervée je suis en colère [car il y a des colères qui sont saines quand elles procèdent d'un sentiment de révolte devant l'injustice] ». Les animateurs tentent par trois fois, mais sans grand succès, de fermer cette parenthèse et de lancer le thème de l'Europe. Au moment même où il semble qu'ils $y$ soient enfin parvenu, Sarkozy revient machiavéliquement à la charge :

NS : non: (.) pis je (.) et je vais même vous dire quelque chose [...] je vous en veux pas parce que ça peut arriver à tout le monde de s'énerver\}

et c'est reparti pour un dernier tour, car SR ne peut une fois encore que protester « je ne m'énerve pas je me révolte car j'ai gardé ma capacité de révolte intacte ", mais ce définitif « mot de la fin » se trouve noyé dans un brouhaha général. En un tournemain, elle change alors à la fois de ton, de thème et de posture pour se lancer, après une sorte de petit rire qui marque ce radical changement de footing, dans un discours sur l'Europe car n'est-ce pas, l'Europe « c'est important » :

SR : non [je je ne m'énerve je ne m'énerve pas je me révolte $\backslash()$.

PPDA : [alors comment vous allez faire l'un et l'autre pour relancer [la machine (.)

européenne (.) [s'il vous plaît

NS : [la question de l'Europe

AC : [(s'il)

SR : \&[car j’ai gardé (.) [ma capacité (.) de révolte (.) intacte $\backslash$

AC : [madame Royal (.) l'Europe

PPDA: [pardonnez-nous de ne poser que des questions mais (.) l'Europe c'est vraiment important [[voilà 
AC : [ [l'Europe c'est important (.) comment\&

$\mathrm{SR}:[[$ (rires) (c'est important)

AC : \&relance-t-on la machine européenne euh [la France\&

$\mathrm{SR}:[\mathrm{hm} \mathrm{hm}$

Sans entrer dans le détail de l'analyse de cette séquence, on se demandera quels enseignements on peut en tirer par rapport à la question qui nous occupe ici.

\subsubsection{Texte, cotexte, contexte}

(1) Le texte global, qui au cours de l'analyse est décomposé en segments successifs, c'est l'ensemble du matériel sémiotique (verbal, paraverbal et non verbal) produit par les participants "actifs ». Il est à peu près le même pour tous les « membres » - à peu près car les personnes présentes sur le plateau ont un accès visuel à ce qui s'y passe plus complet que les téléspectateurs, soumis aux contraintes du cadrage imposé par l'équipe de tournage, elle-même tenue de respecter certaines règles comme on l'a vu plus haut. En outre, si le texte est le même pour tout le monde dans sa matérialité, le texte effectivement " traité » n'est pas exactement le même étant donné que le régime d'écoute qui est appliqué à cet objet matériel va varier d'un auditeur à l'autre (celle des téléspectateurs étant dans l'ensemble plus «flottante » que celle des participants actifs, et celle des animateurs ne se focalisant pas sur les mêmes points que celle des débatteurs, sans parler des cameramen qui s'emploient surtout à guetter les signes anticipateurs d'un changement de locuteur...).

D'autre part, on peut admettre que la compétence sémiotique nécessaire au décodage de ce matériel (savoirs sur la langue et sur les unités mimo-gestuelles qui accompagnent la production des énoncés) est elle aussi à peu près partagée par tous - encore que les systèmes lexicaux des deux principaux protagonistes ne coïncident pas tout à fait puisque NS assimile l'énervement et la colère alors que SR oppose l'énervement à la " saine colère " (syntagme qu'elle s'emploie à construire et définir tout au long de la séquence). On assiste donc en direct à la négociation conjointe du sens et du référent des mots manipulés : «énervé » et «en colère » sont-ils synonymes? Et quelle est la vraie nature de l'état émotionnel de SR ? comment le catégoriser? Cette négociation se fait certes à la marge du lexique: on ne peut pas faire dire n'importe quoi aux mots, qui ont un noyau dur et résistant. Mais ils ont aussi des bords flous, et c'est sur ces bords que tout se joue, car l'enjeu est de taille : ce n'est rien moins que la « présidentiabilité » de Ségolène qui est en cause dans ce débat sémantique, NS répétant que " pour être président de la république faut être calme » quand SR rétorque qu'au contraire, un bon président doit être capable de se mettre en colère devant le spectacle de l'injustice et de l'immoralité politique.

(2) Le cotexte, c'est le matériel sémiotique qui précède et suit la séquence soumise à l'analyse, au sein de la même macro-unité (en l'occurrence l'ensemble du débat) ainsi qu'on l'a vu plus haut. Le cotexte antérieur peut varier d'un téléspectateur à l'autre en fonction du moment où il se branche sur l'émission; quant au cotexte postérieur, on a vu qu'il n'était pertinent que pour l'analyste, qui travaille "après coup». On a vu aussi (exemple de la « cage d'écureuil») que certains éléments du texte pouvaient renvoyer à des segments discursifs extérieurs à cette unité, et qui relèvent de l'intertexte. Ces échos peuvent être plus ou moins lointains ou proches. Par exemple, le syntagme "saine colère " peut faire penser à des expressions similaires telles que les raciniens «juste courroux » ou « courroux légitime » (Andromaque : « Poursuivez, s'il le faut, un courroux légitime »), cette lexie plus ou moins inédite devant elle-même être abondamment reprise 
à la suite de Royal. Mais notre séquence comporte un autre phénomène intertextuel intéressant : il s'agit du moment où Sarkozy déclare à sa rivale :

donc j'ai le droit de parler du handicap (.) ce n'est pas le monopole (.) qui est le vôtre (.)

déclaration dont la formulation quelque peu bancale fait irrémédiablement penser au fameux « Vous n'avez pas le monopole du cœur » lancé à Mitterrand par Giscard d'Estaing dans un contexte similaire, puisqu'il s'agit du débat pour le deuxième tour des présidentielles de 1974. Or il se trouve que le 15 janvier 2007, lors d'une discours prononcé au Mont Saint-Michel Sarkozy avait eu ce lapsus peut-être pas révélateur mais à coup sûr malencontreux : « il y a quelques années, François Mitterrand dans une réplique superbe avait dit: vous n'avez pas le monopole du cœur». On peut donc être tenté d'interpréter la phrase prononcée par NS face à SR, peu de temps après cet incident, comme une sorte de contournement de la formule sur «le monopole du cœur», qui aurait tout à fait sa place ici, mais qui rappelle à NS un souvenir trop cuisant pour qu'il ose la ressortir : c'est tentant, mais risqué - il l'ébauche donc, mais sans aller jusqu'au bout de la formule. Du point de vue de l'interprétation cet exemple est particulièrement intéressant car le mécanisme intertextuel a bien un support inscrit dans la matérialité discursive : c'est d'une part le mot «monopole » et d'autre part le fait que la phrase «ce n'est pas le monopole qui est le vôtre » a quelque chose qui cloche. Mais c'est un support discret, qui suggère simplement une interprétation du genre de celle qui vient d'être proposée, et seulement à ceux qui possèdent cette double information - celle de 1974 est très largement partagée, celle de janvier 2007 l'est beaucoup moins; elle est bien évidemment connue de NS lui-même, sans que l'on puisse cependant être sûr qu'elle lui soit consciemment revenue en mémoire à ce moment du débat avec Royal. Tout cela pour rappeler que la question de la description « du point de vue des membres » soulève la question préalable du «quels membres?» Qui sont les récepteurs susceptibles de percevoir cette double référence intertextuelle? NS et SR? OM et AC? Quelques téléspectateurs particulièrement avisés et vigilants? L'interprétation de ce segment discursif va varier en fonction des connaissances préalables dont disposent les « membres » mais aussi de leur capacité à les mobiliser le moment venu, qu'il s'agisse des savoirs intertextuels ou contextuels, qui sont en permanence injectés dans les énoncés pour permettre leur interprétation.

53 (3) Le contexte englobe une infinité d'éléments dont certains sont immédiatement accessibles, comme tout ce qui relève de l'apparence physique des participants ${ }^{14}$, et qui est à coup sûr pertinent au même titre que l'ensemble de leur comportement - en particulier, le fait que l'un(e) des candidat(e)s soit « visiblement $»^{15}$ une femme ne peut pas ne pas avoir d'incidence sur le comportement de son adversaire. On peut par exemple noter que la gestuelle de Sarkozy face à Royal est remarquablement « douce " par rapport à celle qu'il mobilise face à Le Pen dans le débat précédemment évoqué : il remballe son poing fermé, signe de pugnacité virile, et même son geste préféré, l'index pointé accusateur, qui se trouve en quelque sorte " mis à l'index » alors que Royal, elle, n'hésite pas à y recourir dans le réquisitoire qui ouvre la séquence de la saine colère ; geste que d'ailleurs Sarkozy ne manque pas de lui reprocher, de manière assez cocasse : « calmezvous et ne me montrez pas du doigt avec cet index pointé parce que franchement... ». Mais malgré les efforts que déploie NS pour ne pas paraître agressif ni sexiste, il est inévitable que l'accusation de "perdre ses nerfs " qu'il porte contre son adversaire féminine se charge d'une connotation spécifique, celle de la «femme hystérique ». De 
même, lorsqu'au terme de l'émission les animateurs annoncent à NS qu'il a encore droit à trois minutes alors que SR a épuisé son temps de parole, NS déclare de façon chevaleresque qu'il «rend ces trois minutes à madame Royal»; mais il ajoute moins chevaleresquement :

NS : [mais bien sûr je rends bien volontiers ces trois minutes à ma-madame Royal/

moi je veux être précis concret (.) et je je n'juge pas ça :(.) au à la quantité

où l'on voit se profiler le topos de la «femme bavarde », qui parle d'abondance mais pour ne rien dire. Comme quoi un même discours ne sonne pas exactement de la même manière selon qu'il est adressé à une femme ou à un homme, du fait de l'existence de certaines idées reçues qui rôdent dans le contexte, toujours prêtes à se projeter sur le texte pour créer certains « effets de sens » bien particuliers.

Si certaines données contextuelles, comme le sexe des participant(e)s, sont fournies dans l'interaction elle-même, la plupart des constituants pertinents du contexte relèvent de savoirs préalables, concernant entre autres : les participants au débat et leur « éthos », ce qui crée un certain nombre d'attentes avec lesquelles les débatteurs vont jouer, en les déjouant à l'occasion, comme dans ce débat où tous les commentateurs ont signalé que Sarkozy avait «mis un bémol à sa rhétorique » en affichant plutôt un "profil bas »; le type d'événement communicatif engagé, avec ses règles et ses enjeux; et au-delà, le fonctionnement de notre système politique et électoral... Une petite partie de ces éléments contextuels se trouve inscrite dans le texte sous forme de "formulations » (au sens que l'analyse conversationnelle donne à ce terme) telles que "nous débattons ", "quand je serai présidente de la république ", «si je suis élu », etc. Ces formulations sont autant d'inscriptions du cadre de l'interaction dans son texte même, mais elles ne sont interprétables qu'à partir de savoirs externes que l'on convoque en permanence au fur et à mesure que le texte défile sous nos yeux. Or ces savoirs sont éminemment variables au sein de la communauté extrêmement composite des "membres", en ce qui concerne surtout le vaste ensemble des téléspectateurs, qui pose à l'analyste un double problème : celui de leur hétérogénéité, et celui de l'impossibilité où l'on se trouve d'accéder à leur " point de vue ». L'existence de cette couche de récepteurs, que l'on ne peut pourtant pas exclure de la communauté des "membres» sous prétexte qu'ils ne participent pas activement au débat, met en déroute le postulat qui fonde le travail descriptif en analyse conversationnelle, à savoir que les membres rendent "publiquement disponible » par leur comportement la façon dont ils interprètent ce qui se passe. Car on ne peut avoir accès que de façon très partielle, indirecte, et différée (par le biais de sondages, d'interviews, de commentaires dans la presse ou sur Internet...) aux réactions extrêmement contrastées de ces millions de récepteurs qui sont en réalité les principaux destinataires du petit drame qui se joue sur le plateau de la télévision.

\subsubsection{La tâche de l'analyste}

Il revient d'abord à l'analyste de décrire ce qui se passe " vraiment », en portant attention à tous les détails du texte de l'interaction (car à la différence des participants, il a tout son temps! son traitement du texte est donc maximal, alors que celui qu'effectuent les membres est nécessairement, à des degrés divers, partiel et sélectif). Dépassant la simple paraphrase (NS dit X, SR lui répond que Y), cette description doit d'abord effectuer ce que Plantin (1998) appelle un diagnostic argumenté, c'est-à-dire fondé sur l'interprétation raisonnée des données empiriques. Par exemple, l'examen scrupuleux du comportement de Royal tout au long de cette séquence permet de conclure au fait que non, elle n'est pas 
« sortie de ses gonds », et que sa colère est extrêmement maitrisée, comme en témoignent d'abord le fait que cette séquence est soigneusement "cadrée » (annonce solennelle en ouverture, petite rire et changement soudain de posture en clôture) mais aussi l'ensemble de son comportement discursif. L'émotion dite ( «je ne suis pas énervée, je suis en colère ») se trouve en effet confirmée par l'émotion montrée (si tant est que l'on puisse distinguer clairement les marqueurs caractéristiques de la colère et de l'énervement) : si SR attaque fort dès le début avec une prosodie et une gestuelle caractérisées par une violence incontestable (elle « monte sur ses grands chevaux »), on ne constate au cours du déroulement de la séquence aucune gradation particulière ni aucun bredouillement ou autre indice de perte de contrôle : imperturbablement, SR affiche une colère "froide " (conformément à la définition qu'elle nous donne d'elle-même: «je ne suis jamais énervée j'ai beaucoup de sang-froid ») et de bout en bout contrôlée.

Pourtant, à l'encontre de ce diagnostic la grande majorité des commentateurs de cet incident, qu'ils soient profanes ou experts, ont parlé et parlent encore du «moment où Ségolène Royal s'est énervée » (en langage plus familier, elle aurait " pété les plombs »). Or nous pensons que l'analyste n'a pas le droit de traiter par le mépris les interprétations qu'il juge plus ou moins « erronées », mais qu'il se doit au contraire de tenter d'en rendre compte à partir du moment où elles ne sont pas isolées. En l'occurrence, on peut alléguer divers facteurs favorisant cette interprétation. Il y a d'abord le fait qu'à force de marteler «Ne vous énervez pas» NS a fini par convaincre les spectateurs-auditeurs (ses partisans surtout, mais non exclusivement), que SR s'était effectivement énervée. Il y a aussi le fait que le distingo, légitime sans doute, que tente d'établir Royal entre deux affects qui sont tout de même proches est sans doute trop subtil dans un tel contexte (il ne s'agit pas d'un débat philosophique de France Culture): la langue ordinaire admet bien une sorte de synonymie entre le fait d'être " en colère » et celui d'être " énervé »; la position de NS est donc plus conforme au sens commun que celle de SR, plus conforme aussi à la représentation prototypique de la colère qui est plutôt vue comme un affect violent où l'on casse tout (à l'instar de Moïse ou d'Achille). Il y a enfin l'excessive durée de la séquence : Royal s'acharne, répétant ad nauseam " je ne m'énerve pas je suis en colère »et l'on voit bien pourquoi elle refuse de lâcher le morceau: c'est que c'est son éthos présidentiel qui est en jeu. Mais en s'obstinant de la sorte elle suscite l'exaspération du public qui a vraiment envie que l'on passe à autre chose (l'Europe par exemple), et que s'achève cette parenthèse qui donne l'impression de se faire au détriment des problèmes de fond, comme si ce numéro quelque peu factice et théâtral dans lequel Royal joue les pasionarias offusquées n'était qu'une sorte de cache-misère. Il semble donc qu'en se laissant entraîner dans cette trop longue digression psychologico-éthique Ségolène Royal soit bel et bien tombée dans le piège tendu par son adversaire, qui a beau jeu de tirer la morale de cette histoire :

au moins ça a eu une utilité madame (.) c'est que vous vous mettez bien facilement en colère (.) vous sortez de vos gonds avec beaucoup de facilité madame (.) président de la république (.) c'est quelqu'un qui a des responsabilités lourdes (.) très lourdes

Il serait plus aventureux de faire des hypothèses sur le caractère sincère ou non, prémédité ou non (avec l'aide de son équipe de conseillers en communication), de la " saine colère » de Ségolène Royal. Mais à chacun de répondre à la question de savoir jusqu'où il peut s'aventurer dans le maquis de l'interprétation. En tout état de cause, l'analyste est toujours un archi-interprétant qui a (idéalement) pour tâche de procéder à la description la plus « objective » possible de ce qui se passe tout au long du déroulement 
de l'échange, tout en se donnant les moyens de rendre compte de la subjectivité interprétative des différents participants impliqués à quelque titre que ce soit dans l'événement communicatif étudié.

\section{Conclusion}

Soit A qui entre dans une boulangerie et dit à B : «Une baguette s'il vous plaît. » Il n'est pas trop difficile dans un tel cas de distinguer ce qui relève du texte et ce qui relève du contexte. Mais le texte, ce n'est pas seulement l'ensemble du matériel signifiant (la phrase intonée et mimo-gestualisée), ce sont aussi et surtout les significations qui s'y attachent, dont l'extraction exige la mobilisation d'un certain nombre de savoirs, les savoirs sur la langue et sur le monde environnant étant inextricablement mêlés. Décrire le fonctionnement du discours « du point de vue des membres » c'est se demander quels types de savoirs ils mobilisent pour se comprendre (ou ne pas se comprendre) mutuellement, en l'occurrence : ils savent qu'ils se trouvent dans une boulangerie (savoir situationnel), que dans ce contexte le mot «baguette " renvoie à un type particulier de pain (savoir sémantico-référentiel), que dans ce contexte toujours la requête peut se formuler de façon elliptique mais de préférence accompagnée de "s'il vous plaît », et que A doit vouvoyer B (savoirs socio-pragmatiques) - si par inadvertance le client tutoie indûment le commerçant, l'analyste devra signaler cet emploi déviant même s'il n'est pas relevé explicitement par B ; c'est-à-dire qu'il doit être équipé des diverses compétences dont disposent les divers participants à l'échange, afin d'être en mesure de se mettre « dans leur peau ». Si donc la distinction entre données « internes » et « externes » n'est pas une vue de l'esprit (il y a bien des énoncés, et des choses autour), d'un point de vue descriptif la question pertinente se pose en ces termes: quels sont les types de savoirs nécessaires à l'interprétation du segment discursif soumis à l'analyse (savoirs linguistiques, pragmalinguistiques et socio-pragmatiques, génériques, cotextuels et intertextuels, situationnels, encyclopédiques...) ? S'agit-il de savoirs préalables ou construits au cours de l'événement communicatif lui-même, et sont-ils ou non partagés par les différents participants à l'interaction?

À propos de cette question du contexte, on cite souvent le début de l'article fameux de Goffman intitulé « The neglected situation»:

It hardly seems possible to name a social variable that doesn't show up and have its

little systematic effect upon speech behaviour. $(1964: 133)$

61 et l'on ajoute parfois que ces variables contextuelles sont tout à la fois, pour la production comme pour l'interprétation du discours, un ensemble de contraintes et de ressources. Mais ce ne sont que des ressources parmi d'autres, dont l'importance varie en fonction... du contexte justement, et en particulier du type de discours auquel on a affaire - la principale ressource interprétative restant tout de même la connaissance de la « langue », comme nous le rappelions en ces termes, faisant écho à une citation de Kleiber, dans un précédent article (2009 : 28) :

il ne faudrait pas que l'importance bien légitime accordée au contexte sous toutes ses formes aboutisse à la négation de l'existence, antérieure à l'interaction "située », d'un ensemble de règles au premier rang desquelles il y a les règles proprement linguistiques (c'est-à-dire la « langue »). En d'autres termes : ni sousestimation, ni surestimation du rôle du contexte, qui ne crée jamais du discours ex nihilo. 
Quant à savoir si la langue est elle-même, comme l'affirme Mondada (1998: 244), «structurée de façon constitutivement contextuelle» en ce sens que toutes les unités fonctionneraient à la manière des déictiques :

La reconnaissance de l'importance du contexte s'impose lorsqu'on considère que non seulement les usages mais aussi la définition des ressources linguistiques sont structurés de façon constitutivement contextuelle. c'est une question que nous laisserons pour un autre débat, où l'on se demanderait comment peut être reformulée aujourd'hui l'opposition saussurienne entre la « langue » (rebaptisée en termes de "ressources linguistiques») et la "parole » (c'est à dire les réalisations discursives particulières).

Pour l'heure, nous nous contenterons de reprendre le «ni-ni » de Kleiber - ni le Charybde du « sans-contexte » ni le Scylla du « tout-contexte »-, en l'agrémentant de cet aphorisme emprunté à Nathalie Heinich ${ }^{16}$ : «Entre Charybde et Scylla, toujours prendre la tangente $»$.

\section{BIBLIOGRAPHIE}

Anquetil (S.), 2009 : « Données contextuelles et degré de conventionnalité dans les actes de langage indirects ». In Sandré (éd.) 2009, 41-52.

Constantin de Chanay (H.) \& Kerbrat-orecchioni (C.), 2007 : « 100 minutes pour convaincre : l'éthos en action de Nicolas Sarkozy ». In M. Broth et al. (éds) Le français parlé des médias, Stockholm : Acta Universitatis Stokholmiensis, 309-329.

Duranti (A.) \& Goodwin (Ch.) (eds), 1992 : Rethinking Context, Cambridge : CUP.

Goffman (E.) « The neglected situation », American Anthropologist 66-6, 133-137.

Kerbrat-Orecchioni (C.), 1996 : « Texte et contexte », Scolia 6, 39-60.

Kerbrat-Orecchioni (C.), 2001 : Les actes de langage dans le discours, Paris : Nathan (rééd. Colin 2006).

Kerbrat-Orecchioni (C.), 2002 : «Contexte ». In P. Charaudeau \& D. Maingueneau (éds), Dictionnaire d'analyse du discours, Paris : Seuil, 134-136.

Kerbrat-Orecchioni (C.), 2009 : « Le traitement du contexte en analyse du discours en interaction ». In Sandré (éd.) 2009, 11-29.

Kleiber (G.), 1997 : « Contexte, où es-tu ? », Revue de Sémantique et Pragmatique 1, 65-79.

Mondada (L.), 1998 : « Variations sur le contexte en linguistique », Cahiers de l'ILS 11, 243-266.

Mondada (L.), 2006 : « La question du contexte en ethnométhodologie et en analyse conversationnelle », Verbum XXVIII, 2-3, 111-151.

Plantin (C.), 1998 : « La raison des émotions ». In M. Bondi (éd.), Forms of argumentative discourse, Bologne : CLUEB, 32-50. 
Sandré (M.), 2009 : « Quelles limites pour le contexte en analyse du discours? Analyse des interruptions dans les Questions orales au gouvernement : une nécessaire prise en compte du genre du discours ». In Sandré (éd.) 2009, 83-94.

Sandré (M.) (éd.), 2009 : Analyses du discours et contextes. Limoges : Lambert-Lucas.

Schegloff E.A. (1997) : « Whose text ? Whose context? », Discourse \& Society 8(2), 165-187.

Traverso (V.), 2004 : Analyse des interactions : questions sur la pratique, Document de synthèse en vue de l'HDR, Université Lumière Lyon 2.

Numéros de revues :

Scolia 6, 1996 (P. Schmoll éd.), « Contexte(s)».

Verbum XXVIII, 2-3, 2006 (L. Mondada éd.), « La pertinence du contexte. Contributions de l'ethnométhodologie et de l'analyse conversationnelle».

Pratiques 129-130, 2006 (G. Achard-Bayle éd.), « Textes. Contextes ».

\section{NOTES}

1. Voir Kerbrat-Orecchioni 1996, 2002 et 2009.

2. On peut noter la tendance, dans les universités, à préférer à la veille des grandes vacances « Bon été » à « Bonnes vacances », formule risquée car l'on n'est jamais sûr que le destinataire ait l'intention de prendre de "vraies» vacances étant donné les obligations de recherche qui l'accablent, alors que la venue de l'été est garantie du moins au sens propre du terme, même si B peut toujours rétorquer que l'an passé il n'y pas eu vraiment d'été, d'ailleurs il n'y a plus de saisons...

3. La formule «Bon courage » suppose que l'on ait du courage, mais aussi que le courage soit nécessaire à la réalisation de l'action ou de l'activité projetée: cette expression est donc révélatrice d'une certaine représentation négative de cette activité. La relative fréquence de cette formule votive dans notre environnement quotidien serait donc révélatrice d'une «sinistrose" ambiante, d'après un lecteur s'exprimant en ces termes dans le Courrier de Télérama (3 juin 2009) : "L'avez-vous remarqué ? En prenant congé aujourd'hui, les gens ne disent plus 'au revoir', 'à bientôt', etc., mais ils vous apostrophent d'un définitif 'Bon courage !' $Y$ en a marre de la sinistrose, ne pourrait-on pas dire: 'Au plaisir', 'A la prochaine', 'Bonne journée'?»

4. Voir par exemple Anquetil 2009 sur les demandes d'information.

5. Le pointeur est en l'occurrence Nicolas Sarkozy (voir Constantin de Chanay \& KerbratOrecchioni $2007: 317-318$ ).

6. Voir Kerbrat-Orecchioni $2001: 110-122$.

7. Dans l'édition de 1974 il était question de mot, de phrase, et de «fragment d'énoncé ». Il est permis de voir dans le remplacement de cette expression par le vague mais prudent terme de « passage» la prise en compte du fait que l'analyse linguistique peut s'intéresser à des unités supérieures à l'énoncé.

8. ASP signale une aspiration audible.

9. En particulier du fait d'une généralisation récente de la salutation comme ouvreur obligatoire en toute circonstance, même dans des cas où naguère, une excuse ou un simple sourire suffisaient. Si l'on «oublie» ainsi de saluer quelqu'un que l'on aborde (pour demander une renseignement par exemple), on risque de se voir décocher un «Bonjour ! fortement intoné qui sonne comme un petit coup de semonce. 
10. Ce traitement s'oppose à celui de Gumperz, pour qui les marqueurs prosodiques sont des "indices de contextualisation», qui viennent se surajouter aux marqueurs proprement linguistiques pour en infléchir l'interprétation.

11. Cf. Duranti \& Goodwin (1993: 3) définissant le contexte comme « a frame that surrounds the event being examined and provides ressources for its appropriate interpretation. »

12. Mondada reprend aussi $(2006: 123$, n. 1) un raisonnement de Schegloff baptisé «paradoxe de la proximité » et que l'on peut trouver assez étrange :

«Si un élément du contexte 'externe' est démonstrablement pertinent pour l'organisation 'interne' de l'interaction, alors il perd son statut 'externe'; si sa pertinence ne peut être démontrée, alors son statut de contexte ne peut être invoqué »

- mais si un fait "externe» a des conséquences "internes", cesse-t-il du même coup automatiquement d'être externe? S'il fait froid et que je mets un pull, la froidure est démonstrablement pertinente pour mon action, mais elle ne perd pas pour autant son statut de réalité externe...

13. La transgression délibérée de ce principe produit immanquablement certains effets spéciaux : on peut penser au cas de la « cantatrice chauve » éponyme de la pièce de Ionesco, dont on se saura jamais qui elle est ; c'est le même procédé qu'utilise, plus systématiquement encore, Tardieu dans une piécette intitulée justement Eux seuls le savent - " eux ", c'est-à-dire les personnages, les spectateurs/lecteurs étant quant à eux laissés dans la plus totale ignorance des tenants et aboutissants des événements apparemment fort dramatiques qui se déroulent sous leurs yeux.

14. Alors que la mimo-gestualité relève pour nous du texte de l'interaction, les éléments dits "statiques» sont considérés comme relevant du contexte; mais cette frontière est bien évidemment discutable.

15. Allusion à cette déclaration de Ségolène Royal elle-même lors du débat télévisé du 17 octobre 2006 pour la désignation du/de la représentant(e) du PS à l'élection présidentielle : « Ce qui fait ma différence, c'est aux autres de le dire, [...] en tout cas il y en a une qui est visible, sur laquelle je n'insisterai pas. »

16. Dans Le bêtisier du sociologue, Paris, Klincksieck, 2009.

\section{RÉSUMÉS}

Dans cette contribution nous reviendrons sur un certain nombre des problèmes que pose la notion de contexte, envisagée dans une perspective pragmatique et interactionnelle. À partir de l'exemple des actes de langage (et en particulier du vœu), on montrera que leur description « en langue " nécessite la prise en considération de certains éléments du contexte, cette prise en compte étant a fortiori plus indispensable dès lors que l'on travaille « en discours ", pour décrire l'émergence de certains effets de sens particuliers. À partir de l'exemple d'échantillons de débats médiatiques, on centrera la réflexion sur le fait que le contexte (envisagé sous ses différents aspects) n'est pas le même pour les différents participants à l'événement communicatif et pour l'analyste chargé d'en rendre compte, et l'on se demandera en quoi consistent donc les tâches de l'analyste confronté à la diversité des points de vue sur cet événement.

In this paper we'll come back to some problems raised by the notion of context, considered in a pragmatic and interactional perspective. From the case of speech acts (especially wishes), we'll 
see that their description in the linguistic system requires to take into account some elements of context, which is still more indispensable when dealing with samples of discourse, in order to describe the emergence of some particular " effets de sens ». From the case of mediatic debates, we'll focus on the fact that context is not the same for the different participants to the speech event and for the analyst who has to describe it. Finally we'll raise the question of what the tasks of the analyst, confronted to such diverse points of view, « ideally » consist in.

INDEX

Mots-clés : actes de langage, débats médiatiques, interaction, interprétation, vœu

Keywords : interaction, speech acts, interpretation, media debates, wishes

\section{AUTEUR}

\section{CATHERINE KERBRAT-ORECCHIONI}

ICAR, université Lumière Lyon 2 